$$
E-18-\times 85
$$
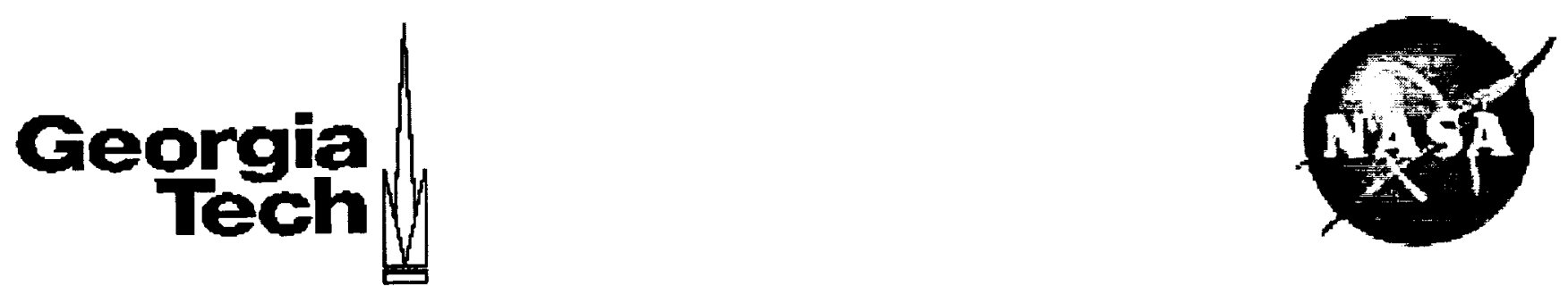

\title{
FATIGUE DAMAGE MECHANISMS IN ADVANCED HYBRID TITANIUM COMPOSITE LAMINATES
}

\section{W. Steven Johnson and Donald W. Rhymer}

School of Materials Science and Engineering

Georgia Institute of Technology

Atlanta, Georgia 30332-0245

Final Report to:

NASA Langley Research Center

Mail Stop 226

Hampton, VA 23681

ATTN: Dr. Terry L. St.Clair, Technical Officer

NASA Grant NAG-1-1890

GT Project \#: E-18-x85 


\title{
FATIGUE DAMAGE MECHANISMS IN ADVANCED HYBRID TITANIUM COMPOSITE LAMINATES
}

\author{
Donald W. Rhymer' ${ }^{1}$ and W. Steven Johnson ${ }^{2}$ \\ School of Materials Science and Engineering \\ George W. Woodruff School of Mechanical Engineering \\ Georgia Institute of Technology \\ Atlanta, Georgia 30332-0245
}

\section{ABSTRACT}

Hybrid Titanium Composite Laminates (HTCL) are a type of hybrid composite laminate with promise for high-speed aerospace applications, specifically designed for improved damage tolerance and strength at high-temperature $\left(350^{\circ} \mathrm{F}\right.$, $177^{\circ} \mathrm{C}$ ). However, in previous testing, HTCL demonstrated a propensity to excessive delamination at the titanium/PMC interface following titanium cracking. An advanced HTCL has been constructed with an emphasis on strengthening this interface, combining a PETI-5/MM7 PMC with Ti-15-3 foils prepared with an alkaline-perborate surface treatment. This paper discusses how the fatigue capabilities of the "advanced" HTCL compare to the first generation HTCL which was not modified for interface optimization, in both tension-tension $(R=0.1)$ and tension-compression ( $\mathrm{R}=-0.2)$.

The advanced HTCL under did not demonstrate a significant improvement in fatigue life, in either tension-tension or tension-compression loading. However, the advanced HTCL proved much more damage tolerant. The $R=0.1$ tests revealed the advanced HTCL to increase the fatigue life following initial titanium ply damage up to $10 \mathrm{X}$ that of the initial HTCL at certain stress levels. The damage progression following the initial ply damage demonstrated the effect of the strengthened PMC/titanium interface. Acetate film replication of the advanced HTCL edges showed a propensity for some fibers in the adjacent PMC layers to fail at the point of titanium crack formation, suppressing delamination at the Ti/PMC interface. The inspection of failure surfaces validated these findings, revealing PMC fibers bonded to the majority of the titanium surfaces. Tension compression fatigue $(R=-0.2)$ demonstrated the same trends in cycles between initial damage and failure, damage progression, and failure surfaces. Moreover, in possessing a higher resistance to delamination, the advanced HTCL did not exhibit buckling following initial titanium ply cracking under compression unlike the initial HTCL.

\footnotetext{
' Graduate student, School of Mechanical Engineering, Georgia Institute of Technology, Atlanta, GA 30332-0405

${ }^{2}$ Professor, School of Materials Science \& Engineering and Mechanical Engineering, Georgia Institute of Technology, Atlanta, GA 30332-0245
} 


\section{INTRODUCTION}

In recent years aerospace researchers have endeavored to meet the ongoing demands of both military and commercial aircraft to fly faster and longer then ever before. Such advancement is contingent upon structural materials capable of operating at long lives ( $>10,000$ hours) and higher temperatures while remaining fatigue resistant and damage tolerant. Hybrid Titanium Composite Laminates (HTCL) are a type of hybrid composite laminate designed specifically for this purpose, possessing superior fatigue resistance and damage tolerance at temperatures up to $350^{\circ} \mathrm{F}$.

Hybrid composite laminates consist of varying layers of metal alloy alternately bonded with layers of polymeric matrix composites (PMC) to produce a high-performance, fatigue-resistant material, retaining the high strength gains of fiber composites without incurring a significant weight penalty. HTCL (also known as Titanium-Graphite Hybrid Laminates, or TiGr) is designed with metal layers of titanium for high temperature durability.

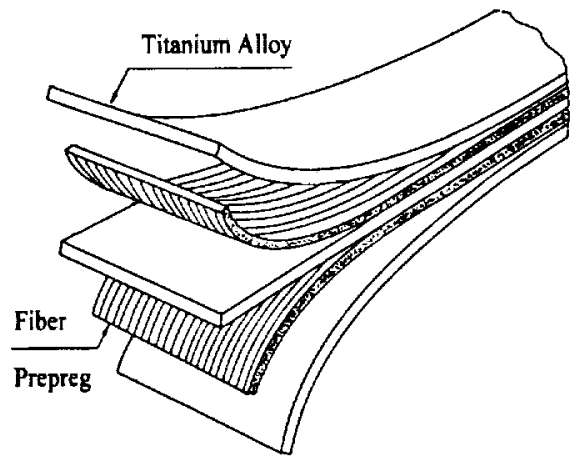

Figure 1. HTCL

The development of hybrid composite laminates originated following the success of extensive lamination research. In 1967, J. Kaufman [1] proved that a laminate of adhesively bonded aluminum plies has nearly twice the fracture toughness of a single aluminum plate of the same overall thickness. In 1978, W. S. Johnson, et al. [2] demonstrated that laminated aluminum greatly improves the fatigue and crack growth resistance of monolithic aluminum. Johnson [3], in 1983, proved these increased damage tolerant properties also characterized adhesively bonded titanium. By the mid-1980's, Delft along with ALCOA developed the first hybrid composite laminate called ARALL. [4] The material is composed of thin aluminum laminae with aramid fiber to reinforce the bondline. The premise and result of the design was to retain the damage tolerance and fracture toughness demonstrated in metal laminates, while incorporating the mechanical advantage the 
fibers offer to strengthen the material and contribute to crack growth resistance of the metal. After the initial success of ARALL, GLARE was developed in 1991, which uses R and S2 glass instead of aramid as the fiber [5]. These materials useful in many designs and are now flying on several commercial and military aircraft.

But in order to meet the requirements of high-speed, high-temperature $\left(177^{\circ}\right.$ C) flight, Johnson, Miller, and colleagues [6] in the mid-1990's developed HTCL, the first temperature resistant hybrid composite laminate. With the preliminary testing proving successful, subsequent, extensive testing was started by E. Li [7] and Johnson in 1996 to demonstrate HTCL's fatigue resistance at elevated temperatures. However, the results showed that HTCL consistently debonded at the titanium/polymer interface. When a fatigue crack forms in a titanium layer, damage evolves quickly by delamination between the layers.

In order to limit such damage, efforts began to determine ways to increase the strength of the titanium/PMC interface. Cobb and Johnson [8] conducted research on the integrity of the bonding between the two to be incorporated in a second-generation, advanced HTCL. Titanium cracked lap shear specimens were tested with combinations of two different polymeric resins with three different commercially available titanium surface treatments in a variety of exposure environments. FMx5 polyimide adhesive bonded with Boeing's Sol-Gel surface treatment possessed superior fracture toughness and crack growth. Even more important, Cobb's method of interface testing was based on crack growth using the strain energy release rate, which Burianek and Spearing [9] directly related this delamination in HTCL, as with other PMC's.

This paper discusses the fatigue results of HTCL having been optimized using a strengthened ply-to-ply interface based on the optimization results found by Cobb. Using a combination of PETI-5 polyimide (FMx5 being 70\% PETI-5) for the PMC matrix surrounding graphite IM7 fibers and an alkaline-perborate titanium surface treatment (creating a bond similar to Sol-Gel), HTCL was modified before construction. This modified HTCL is tested in tension-tension $(R=0.1)$ fatigue for comparison with the previous research by $\mathrm{Li}[7]$ and tension-compression tests $(\mathrm{R}=-$ 0.2 ) were conducted on both laminates to determine the effect of a strong bondline between plies. For this discussion, the previous extensive testing conducted on HTCL will be dubbed "initial HTCL" while this current research of the material will be referred to as "advanced HTCL".

\section{EXPERIMENTAL PROCEDURE}

\section{Material}

All HTCL used in this study is comprised of 4 plies of Ti-15-3 foil $10 \mathrm{mil}$ thick and 3 plies of PMC 5 mil thick. The Ti-15-3 alloy is $\beta$ metastable that has been solution treated, cold rolled, and heat-treated to form a foil with finitely distributed $\alpha$ precipitates with the $\beta$ grains. It should be noted that while the titanium alloy itself in the advanced HTCL is identical to the initial laminate, the 
initial laminate contained Ti-15-3 foil that was 11 mil thick. The PMC layer consisted of carbon IM7 fibers with approximately 0.6 volume fraction within a PETI-5 matrix. For the titanium surface treatment, prior work conducted by Cobb [8], determined that a Sol-Gel process was optimal for the surface treatment of titanium, particularly when coupled with FMx5. However, it was determined [10] that there was a problem with the long-term durability of the interface when the SolGel was bonded to polyimide (such as FMx5, PETI-5). Moreover, at the time of this research, the Sol-Gel process was still being optimized with an adequate coupling agent. In lieu of these problems, an alkaline-perborate solution was used to prepare the titanium surface, which proved cost-effective, environmentally friendly, and chemically compatible with PETI-5. PETI-5 (phenylethynyl terminated imide oligomer) is a thermomechanically stable polyimide adhesive developed by NASA Langley Research Center [11].
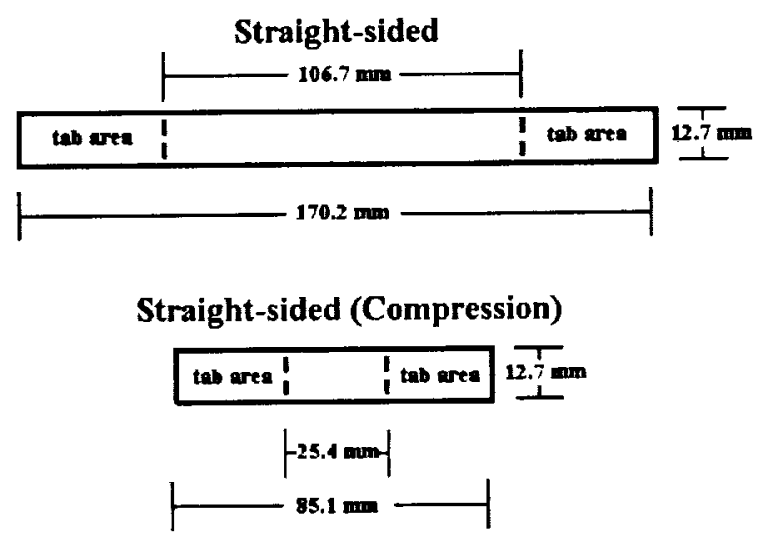

Figure 2. Specimen Configurations

Panels 6.7" X 3.5" of HTCL were autoclave cured and used to cut one of two specimen configurations shown in Figure 2. Long straight-sided specimens were used for tension-tension fatigue testing. Short straight-sided specimens were used for tension-compression fatigue tests. The specimens were tabbed using the same 10 mil Ti-15-3 foil used in the HTCL construction. They were cut to 1.25 " $\mathrm{X}$ 0.5 " and bonded using adhesive film, leaving 4.2" of test length for the long straight-sided specimens and 1.0" of length for the short specimens. Fatigue specimens were polished to a grain size equivalent of $5 \mu \mathrm{m}$ with 4000 grit $\mathrm{SiC}$ paper to better observe damage.

\section{Testing Procedures}

Both tension-tension and tension compression fatigue are conducted in this investigation. The tension-tension tests are conducted for comparison with the previous research conducted on the initial HTCL. Tension-compression fatigue was conducted to more acutely compare the impact of interlaminar strength and 
delamination resistance as compressive stresses are far more detrimental to delamination damage.

Fatigue testing is conducted using a servo-hydraulic test frame with a $25 \mathrm{kN}$ (5.5 kip) load cell and a computer controller/data acquisition system. Servohydraulic pressure grips were used for the testing. An optical microscope was used to detect and photograph HTCL cracks. Delamination was measured by taking impressions using replicating tape and viewing them under the microscope. All tension-tension fatigue tests are conducted using a stress ratio $(R)$ of 0.1 at a frequency of $10 \mathrm{~Hz}$ while tension-compression tests were run with $R=-0.2$ and a frequency of $5 \mathrm{~Hz}$. The frequency is slowed for the compression testing because the damage is expected to propagate much quicker following titanium ply cracking. No change in HTCL fatigue life is expected from this change.

\section{RESULTS AND DISCUSSION}

\section{Constant Amplitude Fatigue}

The results of both tension-tension and tension-compression fatigue will be discussed, beginning with the overall fatigue life followed by a more detailed discussion of the initiation of damage, its subsequent progression, and finally the results of specimen failure investigation.

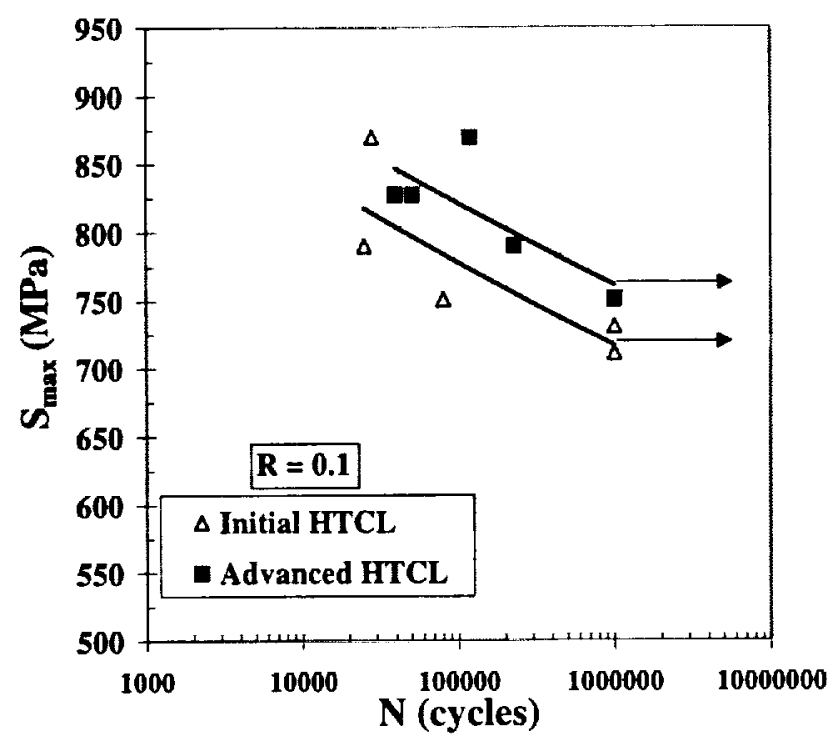

Figure 3 S-N curve comparison between fatigue of initial and advanced HTCL in tension-tension fatigue

The HTCL was fatigued until specimen failure the same stress levels the initial HTCL was previously tested [8]. The specimens were considered failed when they could no longer carry any fatigue load. The number of cycles to failure at 
each stress level was used to produce an $\mathrm{S}-\mathrm{N}$ curve. Figure 3 illustrates the fatigue life for advanced HTCL as compared to initial HTCL. The S-N curves show a similar slope, however the advanced HTCL curve shows higher life with an increase in the endurance limit from $730.8 \mathrm{MPa}(106 \mathrm{ksi})$ to $751.6 \mathrm{MPa}(109 \mathrm{ksi})$. One explanation is due to the difference in titanium ply thicknesses between the two laminates. With $11 \mathrm{mil}$ foil, the initial laminate possesses a volume fraction of PMC plies of .25 while the titanium being .75. In the advanced laminate, the PMC volume fraction is .27 and the titanium is .73. The fatigue loads applied to HTCL are predominantly carried by the much stiffer PMC layer. Therefore, a variation in the percentage of each may alter the fatigue life. Yet this increase in fatigue life and endurance limit is not statistically significant, given scatter and experimental variability. Therefore, little-to-no difference is seen in fatigue life due to the improved interface.

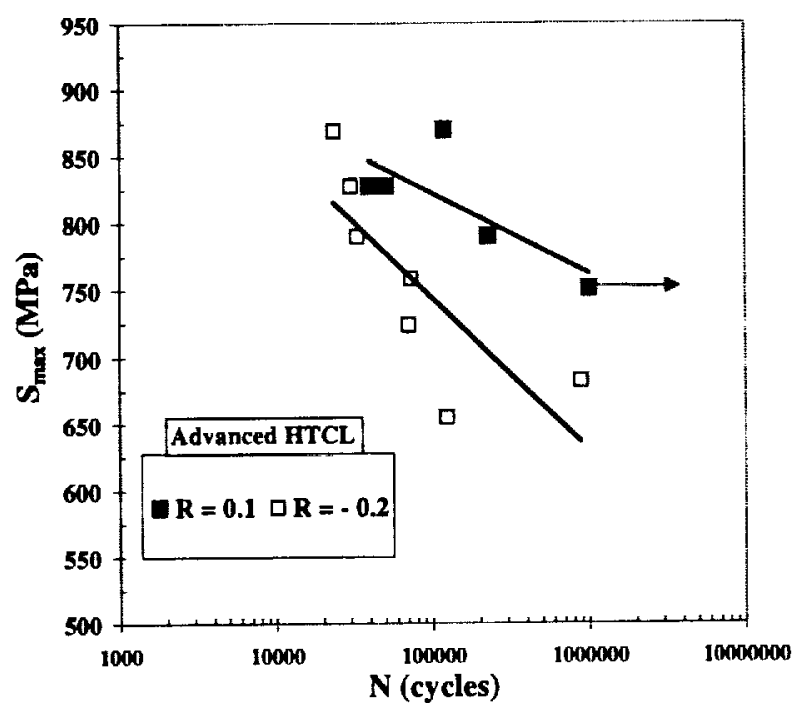

Figure 4 Experimental S-N data comparing advanced HTCL fatigue at $\mathrm{R}=0.1$ to $\mathrm{R}=-0.2$.

Figure 4 shows an S-N curve for the advanced HTCL, using an R-ratio of 0.2 and is graphed in comparison to the tension-tension curve constructed. A slight decrease in fatigue life is shown for the tension-compression. This is a result of the substantial increased stress range. Though the S-N curve shows a somewhat disproportional drop in life at high cycle fatigue, indicated by the change in slope from the $R=0.1$ curve to the $R=-0.2$ curve, it should be noted that considerable scatter is expected at HCF. The same trend was observed in Figure 5 for the initial HTCL comparison. (Note that only two initial HTCL specimens were available for the tension-compression testing, with one tested a second time after being tested at $655 \mathrm{MPa}(95 \mathrm{ksi})$ to runout with no damage.) 


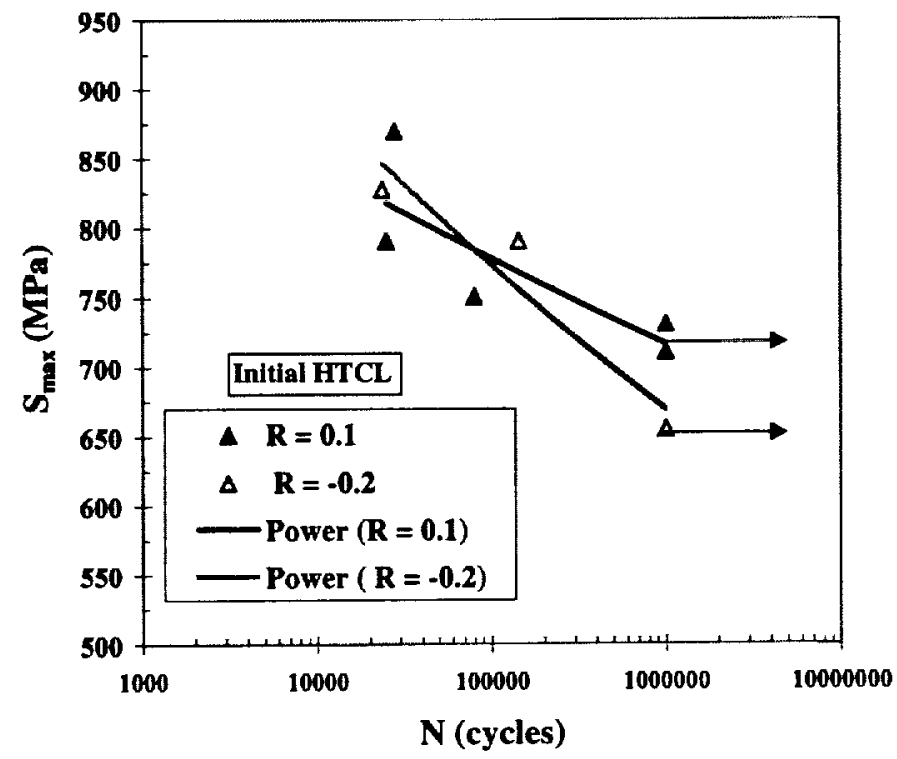

Figure 5 Experimental S-N data comparing initial HTCL fatigue at $R=0.1$ to $R$ $=-0.2$.

\section{Fatigue Damage Initiation}

As expected, the first visible fatigue damage occurs with a crack initiating in a titanium ply. The cracks consistently initiate at one edge and propagate through the entire ply width, a trend Johnson [3] observed in fatiguing adhesively laminated titanium. At the outset of fatigue testing a majority of these cracks initiated in one of the outer titanium plies, as observed in Li's [7] investigation. Table 1 shows the number of cycles from initial titanium cracking to ultimate specimen failure. From these results, it is clear that the advanced HTCL shows a substantial increase in cycles-to-failure following damage initiation over the initial HTCL for identical stress levels. In HCF, the difference is an order of magnitude. Therefore, given titanium ply cracking occurs at the same cycle count for each stress level, the advanced HTCL would maintain a higher, improved S-N curve. This indicates the interfacial strengthening did increase the fatigue life following damage.

As in the case of tension-tension fatigue, tension-compression damage initiated with titanium ply cracking and subsequent propagation across the ply width. Though still the majority of the initial plies to crack were outer plies, inner ply damage occurred initially on a few occasions, as in the case for $\mathrm{R}=0.1$ loading. Table 2 compares the specimen fatigue life remaining following initial titanium cracking for both laminates at $S_{\max }=786 \mathrm{MPa}(114 \mathrm{ksi}), \mathrm{R}=-0.2$. While the advanced HTCL continues fatiguing for 8,000 cycles following initial titanium cracking, the initial HTCL only cycles 1,468. This particular initial HTCL specimen was monitored extensively for damage progression and will be further discussed in the following section, however this result even further discriminates 
the effects of the interface between the laminates. The advanced HTCL increase in fatigue life of following damage validates the observation made between the laminates in tension-tension loading that the strengthened interface does increase the laminates damage tolerance.

Table 1 Number of cycles remaining in initial and advanced HTCL following initial titanium ply failure for tension-tension fatigue $(R=0.1)$

\begin{tabular}{|c|c|c|}
\hline $\mathbf{S}_{\max }(\mathbf{k s i})$ & CYCLES BETWEEN FIRST CRACK AND FAILURE \\
\hline & INITIAL HTCL & ADVANCED HTCL \\
\hline $\mathbf{1 2 6 . 1}$ & 9,000 & 19,682 \\
\hline $\mathbf{1 2 0}$ & NA & 21,345 \\
\hline $\mathbf{1 1 4 . 6}$ & 11,000 & 110,321 \\
\hline \multicolumn{2}{|c|}{ NA (Not acquired) }
\end{tabular}

Table 2 Number of cycles remaining in initial and advanced HTCL following initial titanium ply failure for tension-compression fatigue

\begin{tabular}{|c|c|c|}
\hline $\mathbf{S}_{\max }(\mathrm{ksi})$ & \multicolumn{2}{|c|}{ CYCLES BETWEEN FIRST CRACK AND FAILURE } \\
\hline & INITIAL HTCL & ADVANCED HTCL \\
\hline $\mathbf{1 2 0}$ & NA & 16,700 \\
\hline $\mathbf{1 1 4 . 6}$ & 1,468 & 8,000 \\
\hline
\end{tabular}

\section{Fatigue Damage Progression}

Tension-tension damage propagation following initial titanium cracking was observed for the advanced HTCL, but was not previously accomplished for initial HTCL. The damage was closely monitored to observe any effects of the strengthened interface, specifically the advent of titanium ply delamination due to Mode IMode II (mixed mode) type loading. To do so, the fatigue cycle was stopped once a titanium crack was observed and plastic replications were taken of the edge of the specimen at the area of this crack. Some of the initial cracks occurred in one of the inner titanium plies, which was a different trend from the initial HTCL investigation. 
A case with an inner ply titanium crack initiating first is shown in Figure 6 with a titanium crack forming at 100,000 cycles in a specimen tested with a maximum stress level of $869 \mathrm{MPa}$ (126 ksi). The figure shows a replication of the damage area along with a schematic showing the damage progression. The magnitudes of the damage lines in the schematics are not drawn to scale, however their lengths are reported with dimensions of $\mu \mathrm{m}$.
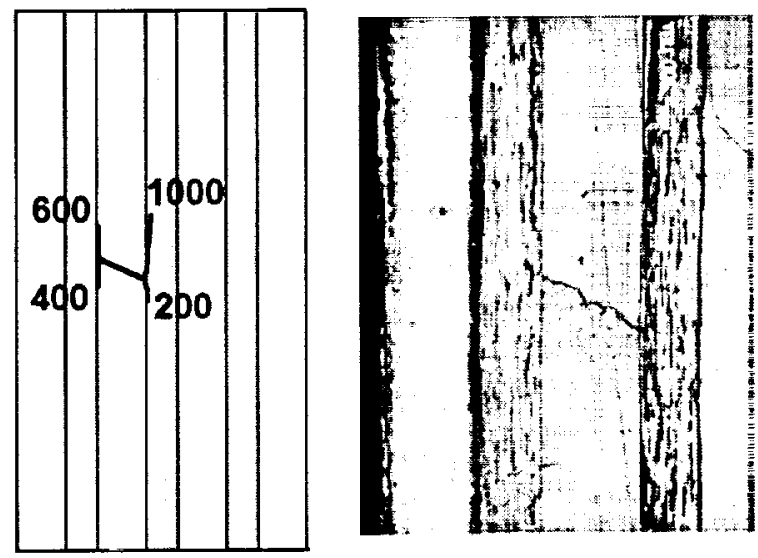

Figure 6 Replication and schematic of HTCL back edge showing fatigue crack and damage after 100,000 cycles. $\left(S_{\max }=869 \mathrm{MPa}, \mathrm{R}=0.1\right)$

A replication was taken of this cracked region on both the front and back edges every subsequent 5,000 cycles until specimen failure. These replications were viewed under the microscope to determine the extent of the damage for each number of cycles. The schematics show the some of the damage to propagate along the interface but some damage, though still mostly following a path in the longitudinal direction, begins to propagate into the PMC layers. Figure 7 shows the adjacent outer ply cracked at 116,000 cycles. The $(+)$ sign shown indicates the damage propagated at least to the magnitude reported beyond as it progressed beyond the area of the replication. Fibers are visibly bonded to the titanium ply along its length. This is a direct result of a strengthened interface

Figure 8 shows a cracked titanium ply in the initial HTCL consistently delaminated along the length of the specimen at the interface. The advanced HTCL possesses a strong enough interface to prevent interfacial delamination, such that a stress concentration breaks the fibers still bonded to the cracked titanium. It is important to note that the first observation only characterized one of two typical scenarios resulting from a failed titanium ply. While often no interfacial delamination was produced between the titanium and PMC layers, in many cases, delamination did occur. However, such delamination always eventually propagated from the interface to the PMC layer, such that most of the length of the failed titanium ply remained bonded to some layers of fiber. This trend is discussed in more detail in the following section as failure surface inspection sheds significant light on this finding. 